\title{
Effects of lag on cued alternation
}

ELLERY S. GORDON and MYMON GOLDSTEIN, Department of Psychology, Long Island University, Brooklyn, N.Y.

Reinforcement was based on position in a two-choice situation and occurred consecutively on the same side until a shift was cued by a change of nonsense form. A lag of $0,1,2$, or 3 display presentations intervened between the change of nonsense form and the shift in reinforcement. Human Ss clearly mastered the Lag 0 and Lag I treatments, but did poorly with the others.

Although a body of literature has been devoted to the topic of systematic positional alternation behavior in animals and humans (Levine, 1963), little is known about regularly alternating positional shifts that depend on an auxiliary cue. The study described below deals with alternation behavior cued by a succession of transient stimuli in a manner suggested by the work of Fleming (1964).

\section{METHOD}

Four versions of an alternation task were related to a single series of 352 displays. Each display contained a pair of identical nonsense forms, and each nonsense form ran consecutively over 4, 5,6 or 7 displays without ever recurring afterwards. The first 22 displays are shown schematically in Table 1, where letters of the alphabet represent nonsense forms like those pictured by Goldstein \& Weber (1965). These 22 displays comprise the first of 16 sets, with each set containing a nearly random arrangement of the four run lengths. The nonrandom feature of the arrangement was avoidance of duplication of run length at any boundary between sets. There were four nonsense forms per set, hence 64 nonsense forms in all. For data handling purposes, adjacent sets were paired to form blocks. There were thus eight blocks, each involving eight nonsense forms and a sequence of 44 displays

Reinforcement was based on position and also involved run lengths of 4 5,6 or 7 consecutive displays. Any correct position remained correct until a shift to the other position was cued by a change of nonsense form. As shown in Table 1 , the shift took effect immediately under the treatment known as Lag 0 , but was delayed for 1, 2 or 3 displays, respectively, under the treatments known as Lag 1, Lag 2 and Lag 3. Note that the latter three treatments required an initial positional run spanning fewer than four displays. It is useful to employ data handling methods from the learning set literature (Harlow, 1959) and distinguish "problems" from "trials within problems." Present purposes will be served by defining a problem as a consecutive run of identically reinforced displays, which implies that Trial 1 of each problem was the trial on which a shift to a new position was supposed to occur.

The 352 displays were presented by means of index cards stacked on arch binders. The Ss served individually and were instructed to turn one card at a time without skipping or looking back. Choices were made by pointing, and E provided reinforcement by saying "correct" or "wrong." There were 10 Ss per lag treatment, making 40 in all. The Ss were undergraduates at Long Island University.

\section{RESULTS AND DISCUSSION}

Each $S$ was given a score representing the number of problems for which Trial 1 was correct. Means were obtained separately for Lags $0,1,2$ and 3 , and had the values 50.1 , $40.9,23.7$, and 23.6 , respectively. Corresponding SDs were $17.5,15.6,9.3$, and 13.0 , respectively. A median test of the differences between treatments yielded a chi-square that was significant at the $2 \%$ level of confidence as a result of a sharp gap between Lags 1 and 2. Similar evidence resulted from a count of Ss who chose correctly on Trial 1 for the last 20 problems. There were 7, 5, 1, and 0 such Ss for Lags 0, 1, 2 and 3 , respectively.

Learning curves for Trial 1 appear in Fig. 1. As before, the data for Lags 2 and 3 are quite similar and indicate that both of these treatments were the most difficult. Lag 0 was easiest. Lag 1 was clearly intermediate at the start but eventually yielded a level of success only slightly lower than that of Lag 0 .

Table 1

Schematic Representation of Experimental Design ( $L=$ Left; $R=$ Right)

\begin{tabular}{cccccc}
\hline & & \multicolumn{4}{c}{ Correct Position } \\
\cline { 2 - 6 } Order & Display & Lag 0 & Lag l & Lag 2 & Lag 3 \\
\hline 1 & a a & L & R & R & R \\
2 & a a & L & L & R & R \\
3 & a a & L & L & L & R \\
4 & a a & L & L & L & L \\
5 & a a & L & L & L & L \\
6 & a a & L & L & L & L \\
7 & b b & R & L & L & L \\
8 & b b & R & R & L & L \\
9 & b b & R & R & R & L \\
10 & b b & R & R & R & R \\
11 & b b & R & R & R & R \\
12 & c c & L & R & R & R \\
13 & c c & L & L & R & R \\
14 & c c & L & L & L & R \\
15 & c c & L & L & L & L \\
16 & d d & R & L & L & L \\
17 & d d & R & R & L & L \\
18 & d d & R & R & R & L \\
19 & d d & R & R & R & R \\
20 & d d & R & R & R & R \\
21 & d d & R & R & R & R \\
22 & d d & R & R & R & R \\
\hline
\end{tabular}




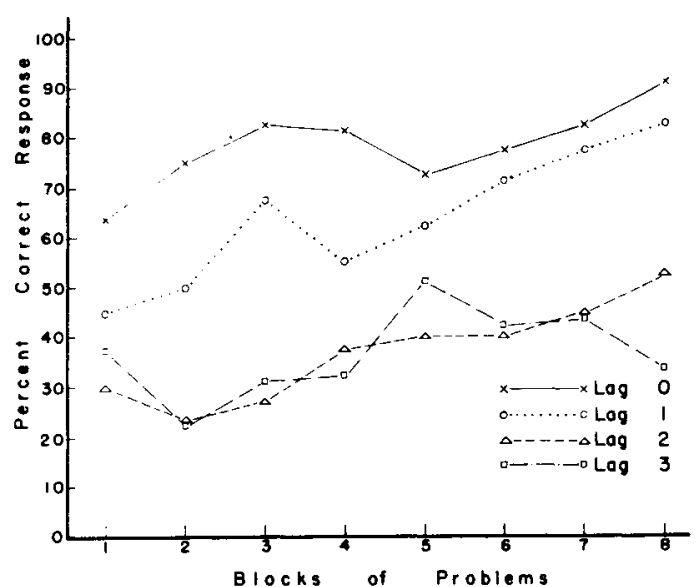

Fig. 1. Learning curves for Trial 1 across problems. There are eight problems per block.

Only Trial 1 exhibited substantial differences between treatments. From Trial 2 onward within problems, all treatments were quite easy, indicating that most Ss who failed to master a change-of-nonsense-form-plus-lag principle achieved partial solutions by learning to choose consecutively on the same side until told that they were wrong. (For a similar observation in a somewhat different situation, see the last paragraph of the paper by Goldstein \& Weber, 1965.) Little can be learned from elaborate treatment of the data not yet covered, but a general appraisal is facilitated by Fig. 2, which contains learning curves for Trial 2. Most learning

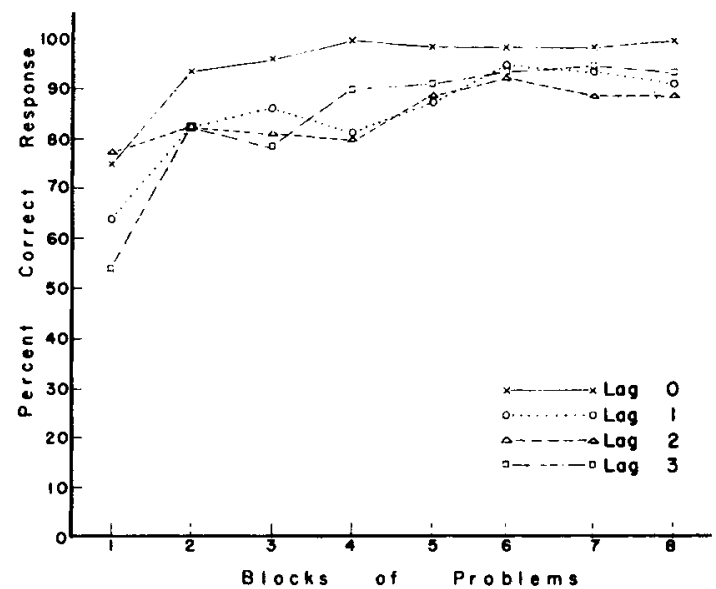

Fig. 2. Learning curves for Trial 2 across problems. There are eight problems per block.

related to Trial 2 occurred at the very beginning, and Lag 0 had a slight edge over the other treatments.

\section{REFERENCES}

FLEMING, J. Discrimination of a pattern of change. Unpublished doctoral dissertation, Princeton University, 1964.

GOLDSTEIN, M., \& WEBER, R. J. Learning set with intra-problem stimulus variation. Perceptual \& Motor Skills, 1965, 21, 799-805.

HARLOW, H. F. Learning set and error factor theory. In S. Koch (Ed.), Psychology: A study of a science. Vol. 2, New York: McGraw-Hill, 1959, Pp. 492-537.

LEVINE, M. Mediating processes in humans at the outset of discrimination learning. Psychological Review, 1963, 70, 254-276. 